\title{
A systematic review and meta-analysis of medium-chain triglycerides effects on acute satiety and food intake
}

Article

Accepted Version

Maher, T. and Clegg, M. E. (2021) A systematic review and meta-analysis of medium-chain triglycerides effects on acute satiety and food intake. Critical Reviews in Food Science and Nutrition, 61 (4). pp. 636-648. ISSN 1040-8398 doi:

https://doi.org/10.1080/10408398.2020.1742654 Available at https://centaur.reading.ac.uk/89667/

It is advisable to refer to the publisher's version if you intend to cite from the work. See Guidance on citing.

To link to this article DOI: http://dx.doi.org/10.1080/10408398.2020.1742654

Publisher: Taylor and Francis

All outputs in CentAUR are protected by Intellectual Property Rights law, including copyright law. Copyright and IPR is retained by the creators or other copyright holders. Terms and conditions for use of this material are defined in the End User Agreement.

www.reading.ac.uk/centaur 
Central Archive at the University of Reading

Reading's research outputs online 
1 A systematic review and meta-analysis of medium-chain triglycerides effects on acute

2 satiety and food intake

3 Tyler Maher ${ }^{1,2}$ and Miriam E Clegg 3

$4{ }^{1}$ Diet and Cardiometabolic Health Research Group, Department of Nutritional Sciences,

5 Faculty of Life Sciences \& Medicine, King's College London, London, SE1 9NH (TM).

$6 \quad$ tyler.maher@kcl.ac.uk

$7{ }^{2}$ Oxford Brookes Centre for Nutrition and Health, Faculty of Health and Life Sciences,

8 Oxford Brookes University, Gipsy Lane, Oxford, OX3 OBP.

$9{ }^{3}$ Department of Food and Nutritional Sciences, University of Reading, Whiteknights,

10 Reading, RG6 6AP (MEC).m.e.clegg@ brookes.ac.uk

12 Corresponding author:

Dr Miriam E Clegg

Department of Food and Nutritional Sciences, University of Reading, Whiteknights, Reading, RG6 6AP (MEC). Ph: 01183788723 Email: m.e.clegg@ reading.ac.uk

Sources of support: TM was supported by an Oxford Brookes University Nigel Groome

Scholarship. There are no conflicts of interest associated with this manuscript

Running head: MCT and satiety: a systematic review

Abbreviations list: Medium-chain triglycerides (MCT), cholecystokinin (CCK), long-chain triglycerides (LCT), body mass index (BMI), pancreatic polypeptide (PP), gastric inhibitory polypeptide (GIP), peptide YY (PYY), glucagon-like peptide-1 (GLP-1). request. 
PROSPERO database: registration number: CRD42018092550.

27

28

29

\section{Abstract}

Research has indicated that consuming medium-chain triglycerides (MCT) may be more satiating than consuming long-chain triglycerides (LCT) potentially causing a reduction in energy intake. However not all studies have demonstrated this acute reduction in energy intake and it has yet to be systematically reviewed. Our main objective was to examine how ingestion of MCT influences energy intake, subjective appetite ratings and appetite-related hormones compared to LCT. Web of Science, MEDLINE, CINHAL and Embase were searched for publications comparing the effect of MCT on appetite (commonly hunger, fullness, desire to eat, and prospective food consumption), appetite-related hormones (pancreatic polypeptide (PP), gastric inhibitory polypeptide (GIP), peptide YY (PYY), glucagon-like peptide-1 (GLP1), neurotensin, leptin, total ghrelin and active ghrelin) and energy intake to LCT. A randomeffects meta-analysis was conducted on studies which examined energy intake.

Seventeen studies (291 participants) were included in the systematic review, of which 11 were included in the energy intake meta-analysis. Synthesis of combined data showed evidence of a statistically significant moderate decrease in ad libitum energy intake after both acute and chronic ingestion of MCT compared to LCT when assessed under laboratory conditions (mean effect size: $-0.444,95 \%$ CI $-0.808,-0.080, p<0.017)$, despite little evidence of any effect of MCT on subjective appetite ratings or circulating hormones.

The current evidence supports the notion that MCT decreases subsequent energy intake, but does not appear to affect appetite. Further research is warranted to elucidate the mechanisms by which MCT reduce energy intake. 
51 Key words: Medium-chain triglycerides, satiety, appetite, energy intake, systematic review,

52

meta-analysis

\section{Introduction}

Overweight and obesity are defined as the accumulation of excess body fat which may lead to impaired health (World Health Organisation 2018). Despite the well-reported risks of increased body fat, including type 2 diabetes, coronary heart disease, some cancers, and stroke (National Health Service 2016), overweight and obesity are still increasingly prevalent. In 2016, more than 1.9 billion adults were overweight globally and 650 million of these were obese; figures which have nearly tripled since 1975 (World Health Organisation 2018). These conditions are caused by a chronic energy surplus from either excessive energy intake or inadequate energy expenditure (Hill, Wyatt, and Peters 2012). It is known that adherence to dietary interventions aiming to reduce bodyweight is low due to feelings of hunger (Franklin et al. 1948), meaning that the target weight loss is not always achieved.

As a result of this, foods with enhanced satiety have gained much attention, both commercially and in research (Hetherington et al. 2013; Chambers, McCrickerd, and Yeomans 2015). Medium-chain triglycerides (MCT) are triglycerides with shorter chain lengths (6-12 carbon atoms long) than 'traditional' long-chain triglycerides (LCT; 12+ carbon atoms long). Due to the shorter chain length of MCT, its consumption results in attenuated release of cholecystokinin (CCK) compared to LCT (Feltrin et al. 2007, 2006; Feinle et al. 2001; Matzinger et al. 2000; French et al. 2000). CCK is involved in lipid-related satiety (McLaughlin et al. 1999), and thus LCT promote satiety via this mechanism. However, MCT are absorbed much quicker than LCT (Marten, Pfeuffer, and Schrezenmeir 2006) which leads to large amounts of $\beta$-oxidation (Bach and Babayan 1982) and the production of $\beta$-hyroxybutyrate (Page et al. 2009); a process which is thought to be anorexigenic (Laeger, Metges, and Kuhla 2010; Scharrer 1999). Studies have shown decreased appetite and subsequent energy intake 
after a preload (Rolls et al. 1988), breakfast (Coleman, Quinn, and Clegg 2016; Kinsella, Maher, and Clegg 2017; Van Wymelbeke et al. 1998) or lunch (Van Wymelbeke, LouisSylvestre, and Fantino 2001) containing MCT. This is not a universal finding however, as some studies have reported no difference in energy intake after meals containing MCT or a control oil (St-Onge et al. 2014; Poppitt et al. 2010), and have even shown increased feelings of hunger after MCT-based meals (Valente et al. 2018).

Clearly, the findings surrounding MCT and satiety are mixed. It is therefore important to systematically determine whether MCT ingestion results in greater satiety and decreased energy intake. Thus, this review aims to assess if there is sufficient evidence to support the hypothesis that MCT can increase satiety in comparison with LCT. Specifically, the objectives are to examine if the consumption of MCT decreases energy intake in subsequent eating episodes, if MCT ingestion favourably alters subjective sensations of appetite (i.e. increased fullness and decreased hunger/desire to eat), and to compile the data on the effects of MCT on circulating hormones involved in appetite regulation.

\section{Methods}

This review is reported according to the PRISMA guidelines (Moher et al. 2009), and is registered in the PROSPERO database (registration number: CRD42018092550).

\section{Search Strategy}

The research question of this systematic review was formulated using PICOS (Population, Intervention, Comparison, Outcome, Setting). The population was defined as adults of healthy status excluding overweight or obesity. The intervention was considered to be any investigation examining medium-chain triglycerides or medium-chain fatty acids on appetite and satiety measures. Outcomes incorporated any measure of appetite (i.e. visual analogue scales), physiological markers of appetite regulation (e.g. PYY, ghrelin) and energy intake measures 
101

102

103

104

105

106

107

108

109

110

111

112

113

114

115

116

117

118

119

120

121

122

123

124

125

(ad libitum meals, diet diaries). There was no restriction to the settings in which studies were conducted.

The databases Web of Science, MEDLINE, CINHAL and Embase were searched for studies in the English language between 1970 and 2018 comprising of all human participants using the strategy ("medium chain triglycerides" AND "satiety" AND "human"). The last search was run on 14 May 2018. Previous systematic reviews were screened to identify relevant subject headings and key words to include within each subject category. Reference lists from the resulting articles were also screened to identify any additional articles. Table $\mathbf{1}$ shows a full list of the specific key words.

\section{Exclusion criteria}

Studies were excluded if they did not examine MCT or medium chain fatty acids (or a product containing either) and subjective measurement of appetite sensations or energy or food intake.

Studies were also excluded if they were conducted in animals, or if they contained individuals outside the age range of 18-70 years, or if they did not include an LCT arm that was matched in calories and composition to an MCT arm.

\section{Data Screening}

Records were screened for duplicates, which were removed. Potential studies were identified by examining all titles and removing those which did not contain reference to MCT and appetite or energy intake by one reviewer. The abstracts of the remaining titles were read, and full text copies were obtained if they still met the initial criteria. Information on the remaining studies after abstract screening was tabulated by one researcher (TM), and both investigators (TM and MC) discussed the inclusion of the studies until a mutual consensus was met. The following information was extracted from the included into a spreadsheet: authors, date of publication, sample size, participant characteristics (age, sex, body mass index [BMI]), study setting, source and amount of MCT, appetite outcome measures and results. 


\section{Quality Checks}

Risk of bias was assessed within the individual studies using the Cochrane Collaboration's Tool (Higgins et al. 2011). Selection bias, reporting bias, performance bias, detection bias, attrition bias and other sources of bias (such as funding etc.) were assessed. Eligible studies were included regardless of risk of bias. Table 2 details risk of all sources of bias for each study.

\section{Meta-analysis procedures}

Due to inconsistent reporting of visual analogue scale data (i.e. presented in a variety of ways, graphical format, as raw data or calculated AUC), and the small number of studies examining appetite hormones (four), only acute ad libitum energy intake data was included in the metaanalysis (either at a single meal or over the course of a whole day). The remaining 11 studies were broken down into 20 subgroups, accounting for studies investigating multiple doses of MCT (Rolls et al. 1988, Stubbs and Harbron 1996) or coconut oil (Rizzo et al. 2016), and for studies with multiple investigations (St-Onge et al. 2014). Energy intake (kJ) was measured at both ad libitum meals and habitual daily intake. Where needed, reported values were converted to $\mathrm{kJ}$ before computation to standardise the units. Meta-analysis software (Comprehensive Meta-Analysis, Version 3, Biostat, Englewood, NJ, USA) was used to conduct a meta-analysis on extracted data. Data inputted included sample sizes, mean energy intake for LCT and MCT trials, their respective SDs, and a correlation coefficient to account for the fact that the included studies were crossover trials $(r=0.940$, calculated from energy intake data from the studies included in the review). The software computed effect sizes for each study, as well as an overall effect size using a random-effects model (DerSimonian-Laird inverse variance approach). Effect size was calculated as the standardised difference in means, which we interpreted to be trivial at $<0.2$, small at $0.2-0.3$, moderate at $0.4-0.8$, and large at $>0.8$, as per Cohen (Cohen 1992). Negative effect sizes indicate decreased consumption in MCT trials/conditions, whereas 
positive effect sizes indicated LCT led to decreased energy intake. Publication bias was assessed utilising funnel plots and by quantifying Egger's regression intercept. A significant regression indicates the presence of a small study effect (Sterne, Egger, and Moher 2011).

\section{Results}

\section{Descriptive}

The database search yielded 4,547 results, which was reduced to 3,517 after the removal of duplicates. After the screening of titles and abstracts, 3,302 were removed. Of the remaining 216 texts, 17 satisfied the inclusion criteria (Figure 1.).

Seven studies were conducted in the UK (Clegg, Golsorkhi, and Henry 2013; Clegg et al. 2012; Coleman, Quinn, and Clegg 2016; Kinsella, Maher, and Clegg 2017; Stubbs and Harbron 1996; Rizzo et al. 2016), two in Australia (Feltrin et al. 2004, 2008), two in France (Van Wymelbeke et al. 1998; Van Wymelbeke, Louis-Sylvestre, and Fantino 2001), two in the US (St-Onge et al. 2014; Rolls et al. 1988), and one each in Italy (Barbera et al. 2000), Sweden (Krotkiewski 2001), Brazil (Valente et al. 2018), and New Zealand (Poppitt et al. 2010). Participants were $28.57 \pm 6.20$ years of age with a BMI of $23.49 \pm 3.42 \mathrm{~kg} / \mathrm{m}^{2}$, and there was an average of $15 \pm$ 8 participants per study (means \pm SD). Participants in one study were classified as 'overweight' according to BMI (M. St-Onge et al. 2014), and were classified as 'obese' in one other (Krotkiewski 2001); all others were in the 'normal' BMI category. There was a total of 291 participants, of which 107 were male and 184 were female. There were 11 acute feeding studies ( Rolls et al. 1988; Clegg, Golsorkhi, and Henry 2013; Coleman, Quinn, and Clegg 2016; Kinsella, Maher, and Clegg 2017; Rizzo et al. 2016; Miriam E. Clegg et al. 2012; M. St-Onge et al. 2014; Valente et al. 2018; Van Wymelbeke et al. 1998; Van Wymelbeke, Louis-Sylvestre, and Fantino 2001; Poppitt et al. 2010), three acute infusion studies (Barbera et al. 2000; Feltrin et al. 2008, 2004), and two chronic dietary intervention studies, of which one examined 
participants three times across all arms of the intervention and quantified habitual daily energy intake (Stubbs and Harbron 1996) and the other was a comparison of independent matched groups (Krotkiewski 2001). One dietary intervention provided all foods consumed by participants in 14-day long manipulations, where the amount of energy from MCT was altered (Stubbs and Harbron 1996), and the other was a very low calorie diet, with either MCT or LCT was incorporated into the low-calorie formula incorporated into the diet (Krotkiewski 2001).

\section{Measures}

Fourteen out of fifteen studies used $100 \mathrm{~mm}$ visual analogue scales to measure subjective sensations of appetite (St-Onge et al. 2014). Ten studies examined energy intake during at least one subsequent ad libitum eating episode after consumption of a meal/preload containing LCT/MCT (Rizzo et al. 2016; Coleman, Quinn, and Clegg 2016; Feltrin et al. 2004; Kinsella, Maher, and Clegg 2017; Poppitt et al. 2010; Feltrin et al. 2008; Rolls et al. 1988; St-Onge et al. 2014; Van Wymelbeke, Louis-Sylvestre, and Fantino 2001; Van Wymelbeke et al. 1998), and one examined daily habitual energy intake after MCT was covertly incorporated into the diet (Stubbs and Harbron 1996). In that study, participants were required to consume all meals in the laboratory, but were allowed to leave and were not required to 'live' in the laboratory; and thus ad libitum daily energy intake was quantified. Three of those also included diet diaries for subsequent energy intake (Coleman, Quinn, and Clegg 2016; Kinsella, Maher, and Clegg 2017; Van Wymelbeke et al. 1998). Four studies examined appetite hormones, including pancreatic polypeptide (PP) (Barbera et al. 2000), CCK (Barbera et al. 2000; Feltrin et al. 2004, 2008), gastric inhibitory polypeptide (GIP) (Barbera et al. 2000; Feltrin et al. 2004), peptide YY (PYY) (Feltrin et al. 2008; St-Onge et al. 2014), leptin (St-Onge et al. 2014), glucagonlike peptide-1 (GLP-1) (Feltrin et al. 2004) and both active and total ghrelin (St-Onge et al. 2014).

\section{Test lipids}


201

202

203

204

205

206

207

208

209

210

211

212

213

214

215

216

217

218

219

220

221

222

223

224

225

The main results of included studies are shown in Table 3. Six studies directly compared MCT to LCT, which acted as a control (Barbera et al. 2000; Clegg, Golsorkhi, and Henry 2013; Feltrin et al. 2004; Rolls et al. 1988; St-Onge et al. 2014; Stubbs and Harbron 1996). Two studies compared MCT and LCT, and also included a low-fat/no-fat control (Feltrin et al. 2008; Krotkiewski 2001; Van Wymelbeke, Louis-Sylvestre, and Fantino 2001). Three studies had multiple fats, including several LCT conditions such as sunflower oil, olive oil and butter (Clegg et al. 2012); olive oil and lard (Van Wymelbeke et al. 1998); and two with another test oil, which was conjugated linoleic acid (Coleman, Quinn, and Clegg 2016) and short-chain triglycerides (Poppitt et al. 2010). Two studies used coconut oil as the source of MCT in the study (Rizzo et al. 2016; Valente et al. 2018), and another study used coconut oil as well as MCT (Kinsella, Maher, and Clegg 2017). For the LCT trials and controls, three studies used sunflower oil (Clegg, Golsorkhi, and Henry 2013; Clegg et al. 2012; Rizzo et al. 2016), two used rapeseed oil (Coleman, Quinn, and Clegg 2016; Kinsella, Maher, and Clegg 2017), two used corn oil (Rolls et al. 1988; St-Onge et al. 2014), one used beef tallow (Poppitt et al. 2010), one used extra virgin olive oil (Valente et al. 2018), one used margarine (Van Wymelbeke, Louis-Sylvestre, and Fantino 2001), one study used an unspecified vegetable oil (Stubbs and Harbron 1996), and three studies (which administered the lipids via infusion and not feeding) used emulsions of oleic and linoleic acid (Barbera et al. 2000), oleic acid (Feltrin et al. 2008), and lauric acid (Feltrin et al. 2004). One study did not specify the LCT used in their study (Krotkiewski 2001). In terms of saturation of LCT, six studies utilised LCT with a mixture of polyunsaturated and monounsaturated acids (Barbera et al. 2000; Rolls et al. 1988; Clegg, Golsorkhi, and Henry 2013; St-Onge et al. 2014; Rizzo et al. 2016; Valente et al. 2018), four used purely monounsaturated fatty acids (Kinsella, Maher, and Clegg 2017; Coleman, Quinn, and Clegg 2016; Stubbs and Harbron 1996; Feltrin et al. 2008), two used mixtures of monounsaturated and saturated fatty acids (Van Wymelbeke, Louis-Sylvestre, and Fantino 
2001; Poppitt et al. 2010), and two studies used multiple sources of LCT; polyunsaturated and monounsaturated (sunflower oil), monounsaturated (olive oil) and saturated (butter) fatty acids (Clegg et al. 2012), and saturated (lard) or monounsaturated (olive oil) (Van Wymelbeke et al. 1998). When accounting for studies that provided multiple doses, the dosage of MCT ranged from $10 \mathrm{~g}$ (Poppitt et al. 2010) to $42.4 \mathrm{~g}$ (Van Wymelbeke et al. 1998), with an average dose of $23.8 \mathrm{~g}$. Three studies provided 10-15 g (Poppitt et al. 2010; Rolls et al. 2004; Rizzo et al. 2016), four provided 20-25 g (Coleman, Quinn, and Clegg 2016; Kinsella, Maher, and Clegg 2017; St-Onge and Jones 2002; Rolls et al. 2004), three provided 30-35 g (St-Onge et al. 2014; Van Wymelbeke, Louis-Sylvestre, and Fantino 2001; Rolls et al. 1988) and one provided 40$45 \mathrm{~g}$ (Van Wymelbeke et al. 1998).

\section{Outcomes}

One out of 11 studies (Feltrin et al. 2004) reported decreased energy intake at an ad libitum meal after MCT compared to LCT, although this was only significant in seven studies (Coleman, Quinn, and Clegg 2016; Feltrin et al. 2008; Rolls et al. 1988; St-Onge et al. 2014; Stubbs and Harbron 1996; Van Wymelbeke, Louis-Sylvestre, and Fantino 2001; Van Wymelbeke et al. 1998). The one study that reported decreased intake after LCT compared to MCT reported a significantly lower energy intake after LCT compared to MCT (Feltrin et al. 2004). The average energy intake at the ad libitum meal in that study after LCT and MCT trials, respectively, was $1,747 \pm 633 \mathrm{~kJ}$ and $4,109 \pm 589 \mathrm{~kJ}$. Five studies out of 14 reported significant differences in appetite ratings, which were decreased hunger and increased satiety after MCT (Krotkiewski 2001), increased fullness after MCT (Kinsella, Maher, and Clegg 2017), increased satiety after LCT (Barbera et al. 2000), decreased hunger but also decreased desire to eat after infusion of MCT (Feltrin et al. 2004), and increased hunger and decreased fullness after MCT (coconut oil) (Valente et al. 2018). Three studies (Rolls et al. 1988; Feltrin et al. 2004; Barbera et al. 2000) reported significant adverse effects, which manifested as gastric 
aching after the MCT drinks ( Rolls et al. 1988), and increased nausea after infusions LCT compared to MCT (Barbera et al. 2000; Feltrin et al. 2004).

Only four studies examined blood parameters in response to the oils which showed LCT led to increased postprandial concentrations GIP, neurotensin, PP (Barbera et al. 2000), CCK (Barbera et al. 2000; Feltrin et al. 2008, 2004), PYY (Feltrin et al. 2008) and GLP-1 (Feltrin et al. 2004). Conversely, one study showed that relative to MCT, LCT led to increased postprandial leptin and PYY, and no effect on GLP-1 or total ghrelin, but active ghrelin concentrations were reduced to a lesser extent after MCT (St-Onge et al. 2014).

\section{Meta-analysis}

Due to high levels of heterogeneity $\left(I^{2}=97.0 \%, Q=333.9, T^{2}=0.355, d_{f}=10\right)$, a random effects model was chosen (Ades, Lu, and Higgins 2005). Effect size for acute ad libitum energy intake ranged from -2.235 to 3.789 . Statistics for each individual study are reported in Supplementary table 1 . There was a statistically significant moderate decrease in ad libitum energy intake after MCT ingestion compared to LCT ingestion (mean effect size: $-0.444,95 \%$ confidence intervals -0.808 to $-0.0 .80, N=11, p=0.017$; Figure 2 ). Sensitivity analysis showed that the removal of each study had only minor effects on overall effect size, and no effect on significance. In order to further examine and specify the effect of consuming MCT on satiety, a sensitivity analysis was conducted by removing infusion studies. This did not alter the direction of significance, but it did increase the level of significance (mean effect size: -0.681 , $95 \%$ confidence intervals -0.950 to $-0.412, N=8, p<0.001)$. More sensitivity analyses were conducted in order to specify the effect of MCT without the influence of coconut oil. Similarly to the removal of infusion studies, removal of the comparison of LCT to coconut oil increased the size of the effect of MCT on energy intake (mean effect size: $-0.529,95 \%$ confidence intervals -0.598 to $-0.460, N=10, p<0.001$ ). The funnel plot (Figure 3.) along with Egger's 
regression intercept showed that there were no small study effects (intercept $=-1.094,95 \%$ confidence intervals: -11.481 to $9.293, p=0.817)$.

\section{Discussion}

\section{Main results}

Prior to this review, MCT had been identified as potentially having more satiating properties than LCT, but studies investigating this are sparse and have found equivocal findings. Understanding how MCT may affect appetite may have implications for weight management, as feelings of hunger are known to the linked to the low rates of adherence commonly seen in dietary strategies (Heymsfield et al. 2007; Franklin et al. 1948). Whereas it is well known that protein is the most satiating of the macronutrients and fat the least, a significant portion of energy in the western diet comes from fat, and therefore methods to increase the satiety response to fat has implications for weight management strategies. The purpose of this review was to examine the appetite responses and energy intake after meals containing either MCT or LCT. It was hypothesised that MCT would increase satiety compared to LCT. The analyses show that MCT suppress energy intake compared to LCT, and this appears to be independent of changes in subjective sensations of appetite and alterations in gut peptide hormones.

\section{Energy intake}

The present meta-analysis showed that nine out of 10 studies reported decreased energy intake at an acute ad libitum meal after ingestion or infusion of MCT, and the only study examining habitual energy intake when MCT was incorporated into the diet also led to decreased energy intake compared to LCT. Whereas the decreased energy intake after MCT consumption wasn't significant in all individual studies, the meta-analysis demonstrated a moderate effect of MCT on energy intake compared to LCT. However, it must be noted that these findings are predominantly limited to the first meal after ingestion of MCT and cannot be extrapolated to 
300

301

302

further meals. More research is needed to elucidate whether compensation occurs in later meals, or if an energy deficit is achieved. One study did incorporate MCT as part of the habitual diet in different MCT:LCT ratios and found that habitual daily intake was lower after the high MCT:LCT ratio period (Stubbs and Harbron 1996). Where this does corroborate the hypothesis that chronic consumption of MCT decreases overall intake; whether this is due to repeat exposure of MCT or a persistent effect is still not known. Furthermore, as only one study to date has investigated chronic MCT consumption and habitual energy intake, these results require validation.

Appetite

Despite reported alterations in energy intake, this appears to have occurred without any reporting of an effect on subjective appetite responses, indicating that MCT suppresses ad libitum energy intake without a concomitant change of feelings of hunger. As aforementioned, this requires further investigation as there is a lack of studies investigating energy intake beyond a single ad libitum meal or a single day. Extraction of subjective sensation data was challenging due to the inconsistent reporting of raw values (i.e. only represented in graphical format), and so these were not included in the meta-analysis. Inspection of the results (Table 3) shows that the majority of studies do not report significant differences in any subjective sensation parameter, and when a difference is reported it is not consistent in all parameters in the study (Barbera et al. 2000; Clegg et al. 2012; Kinsella, Maher and Clegg 2017; Stubbs and Harbron 1996; Valente et al. 2017). The only study to show consistent changes in subjective sensations of appetite incorporated MCT into the diet as part of a very low-calorie diet for 4 weeks (Krotkieski 2001). It is possible that acute feedings of MCT do not alter perceptions of appetite, but repeated exposure may do so.

\section{Mechanisms}


Only four studies examined appetite-related hormones, and so drawing conclusions from these studies is mere speculation; however, secretion of CCK, GIP, PP or GLP-1 appears to be more potent after LCT than MCT. Additionally, another study showed that active ghrelin may be suppressed to a lesser extent than after LCT. MCT have been shown to increase stomach concentrations of acylated ghrelin, as MCT and MCFA are directly used for the acylation of ghrelin (Nishi et al. 2005), which may explain the suppression by LCT. Ghrelin is the only appetite hormone known to stimulate hunger (Wren et al. 2000), whereas CCK, GIP, PP and GLP-1 are involved in promoting satiety and satiation (Gibbs, Young, and Smith 1973; Kissileff et al. 1981; Batterham et al. 2002; Flint et al. 2001; Perry and Wang 2012). Taken together, this implies that MCT exert its anorectic affect through non-hormone mediated mechanisms, however the paucity of data makes this speculation. MCT have been shown to delay gastric emptying (Clegg et al. 2012), despite MCFA being absorbed at a much quicker rate than LCFA (Bach and Babayan 1982). MCT consumption also leads to the production of the ketone body of $\beta$-hyroxybutyrate, which may also be anorexigenic (Laeger, Metges, and Kuhla 2010). Future studies should include these measures in their protocols in order to shed further light on these mechanisms.

The one study that found greater ad libitum energy intake after MCT compared to LCT (Feltrin et al. 2004) compared lauric acid (C12) to decanoic (C10) acid via intraduodenal infusion and observed significant differences in ad libitum energy intake. This was accompanied by greater stimulation of CCK and GLP-1 after infusion of C12. This suggests that the longer chain length is more efficacious at decreasing appetite. It has previously been reported incretin responses to infusions of glucose and lipids are not as pronounced as the response to oral ingestion of glucose (Elrick et al. 1964) or lipids (Lindgren et al. 2011). As such, this makes drawing conclusions from infusion studies difficult. It must also be noted that infusion of C12 induced nausea, which may also explain the decreased ad libitum energy intake. This increased nausea 
was also found after the infusion of LCT but not MCT (Barbera et al. 2000), which also may explain increased satiation scores in that study. Only one other study which examined energy intake reported adverse effects, which were in the form of 'gastric aching' (Rolls et al. 1988), which also may partly explain the decreased ad libitum energy intake after MCT ingestion in that study. In the first study to examine MCT and satiety (Rolls et al. 1988), there was a significant interaction between fatty acid chain length and dosage for gastric aching, suggesting that increased dosage of MCT was linked to stronger adverse effects. However, higher doses have been examined with no adverse effects (Van Wymelbeke, Louis-Sylvestre, and Fantino 2001; Van Wymelbeke et al. 1998), and the authors describe that, despite statistically significant differences, absolute differences were small (3.5 $\mathrm{mm}$ on a $100 \mathrm{~mm}$ scale). Only five of the studies included a rating of nausea (Barbera et al. 2000; Feltrin et al. 2008, 2004; Poppitt et al. 2010; Clegg, Golsorkhi, and Henry 2013), which may also confound the effect observed in our meta-analysis, as (although only two studies reported adverse effects) MCT have been shown to cause GI distress (Jeukendrup et al. 1998; Goedecke et al. 2005) and are generally unpalatable (Clegg 2010; Maher and Clegg 2018).

\section{Methodology}

The dosages of MCT provided in the studies included in this review had a large variation (10 $\mathrm{g}$ (Poppitt et al. 2010) to $42.4 \mathrm{~g}$ (Van Wymelbeke et al. 1998) with an average dose of $23.8 \mathrm{~g}$ ). There did not appear to be a relationship between dose and whether there was an effect on energy intake, as despite the study providing the lowest dose reported no effect (Poppitt et al. 2010), another study found significant effects with all three doses administered in their study; the lowest providing $12.04 \mathrm{~g}$ of MCT (Rolls et al. 1988). Furthermore, the greatest decrease observed after MCT ingestion occurred after $30 \mathrm{~g}$ of MCT was provided in a breakfast and preload study (St-Onge et al. 2014). The optimal dose required to beneficially affect appetite remains elusive. One point that must be taken into consideration is the energy contributed from 
the MCT compared to the decrease in subsequent energy intake it begets. MCT was administered in a variety of ways in the studies in this review, including duodenal infusions (Barbera et al. 2000; Feltrin et al. 2004, 2008), being added to beverages (Coleman, Quinn, and Clegg 2016; Kinsella, Maher, and Clegg 2017; Rolls et al. 1988; St-Onge et al. 2014), a low calorie formula (Krotkiewski 2001), being added to solid meals (Van Wymelbeke et al. 1998; Van Wymelbeke, Louis-Sylvestre, and Fantino 2001; Valente et al. 2018), being cooked into other foods (Clegg et al. 2012; Poppitt et al. 2010; St-Onge et al. 2014), ice cream (Rizzo et al. 2016), and being added into the whole diet (Stubbs and Harbron 1996). One practical limitation that must be considered is the fact that the majority of these studies added the test oils to other foods. however the foods were always kept constant and only fats changed ensuring they were controlled.

\section{Limitations}

There are several limitations to this review and meta-analysis. The main limitation to acknowledge is the fact that one reviewer reviewed all papers, instead of multiple reviewers screening all titles and a consensus being met. Furthermore, studies were initially excluded based on titles alone, instead of a title and abstract screening process. These two limitations mean that incomplete retrieval of records cannot be ruled out. Only 16 studies were included based on our criteria, of which 11 were included in the meta-analysis of energy intake (consisting of 20 subgroups). This highlights the limited data examining the role of MCT in satiety rather than a limitation of this review, however there are methodological differences in the studies included which do need to be acknowledged. Three studies used coconut oil as the means of administering MCT (Rizzo et al. 2016; Poppitt et al. 2010; Valente et al. 2018). One study included in this review examined the effect of MCT to coconut oil as well as a control LCT oil, and reported that MCT resulted in lower energy intake compared to both LCT and 
399

400

401

402

403

404

405

406

407

408

409

410

411

412

413

414

415

416

417

418

419

420

421

coconut oil (Kinsella, Maher, and Clegg 2017). This could be due to the higher concentration of lauric acid ( 50\%) (Denke and Grundy 1992) in coconut oil than in MCT oil (1-3\%) (Bach and Babayan 1982; Clegg 2017). It has been shown that only 20-30\% of lauric acid acts as an MCT, whereas the remainder is packed in chylomicrons as with LCT (Denke and Grundy 1992). This implies that coconut oil may not be a suitable method of examining MCT, and this may have affected the results of the meta-analysis. A sensitivity analysis was conducted by removing the one study investigating coconut oil (Rizzo et al. 2016) and the one subgroup that compared coconut oil and LCT (Kinsella, Maher and Clegg 2017), which led to the effect size to increase; which supports the notion that coconut oil is not as effective as MCT at inducing satiety. Two studies, including the only study that reported increased intake after MCT, administered the oils via infusion and not incorporated into a meal (Feltrin et al. 2008, 2004). We did not specify in our criteria that studies included required to have the MCT in a meal, and thus we decided to include these studies, however, the validity of these studies among other feeding studies could be questioned. Furthermore, the one study that reported an increase intake after LCT (Feltrin et al. 2004) compared lauric acid (C12) to decanoic (C10) acid, which is arguably not MCT compared to LCT due to the absorption of lauric acid, as aforementioned. Removal of these studies did not affect the results of the meta-analysis, and thus they have been kept in in order to better represent the available data. However, similar to studies investigating coconut oil, a sensitivity analysis was conducted by removing the two studies which infused MCT (Feltrin et al. 2004, 2008), and this increased the effect size; meaning the inclusion of infusion studies weakened the effect of MCT on energy intake. From a practical standpoint, this further highlights that consumption of MCT leads to suppressed energy intake compared to LCT. 
422 These limitations should be taken into consideration for future research examining this topic,

423 and also shows the small number of appropriate studies examining the effect of MCT on

424 appetite and energy intake.

425

426 Conclusion

427 The present meta-analysis indicates a moderate reduction in energy intake after consumption

428 of MCT, predominantly at single ad libitum meals, but also total daily energy intake after

429 daily incorporation of MCT into the diet. Whether this reduction persists past the first meal

430 after consumption of MCT remains to be elucidated. The systematic review indicates that

431 there is no effect of MCT on subjective sensations of appetite. Further work is required to

432

confirm the role of appetite hormones in the satiety response to MCT, but there is currently

433

no evidence to suggest a hormonal role of MCT satiety. Due to the small total number of

434

studies, coupled with the fact not all included a feeding component and only four examined

435

hormonal responses to MCT, this paper calls for more studies examining MCT and satiety

436 incorporating these measures, as well as appropriate sources of MCT.

437

438 Acknowledgements

439 T.M. and M.E.C. designed the manuscript, developed the search terms and inclusion and

440 exclusion criteria. T.M. did the searches and both authors screening the final papers. TM did

441 the analysis and wrote the paper. MC had responsibility for the final content.

442 Both authors declare no conflicts of interest. 


\section{References}

Ades, A.E., G. Lu, and J.P.T. Higgins. (2005). The interpretation of random-effects metaanalysis in decision models. Medical Decision Making. 25 (6):646-54.

Bach, A.C., and V.K. Babayan. (1982). Medium-chain triglycerides: an update. American Journal of Clinical Nutrition.

Barbera, R., M. Peracchi, F. Brighenti, et al. (2000). Sensations induced by medium and long chain triglycerides: role of gastric tone and hormones. Gut. 46 (1):32-36.

Batterham, R.L., M.A. Cowley, C.J. Small, et al. (2002). Gut hormone pyy3-36 physiologically inhibits food intake. Nature. 418 (6898). Nature Publishing Group:650-54.

Carroll, J.F., K.A. Kaiser, S.F. Franks, et al. (2007). Influence of bmi and gender on postprandial hormone responses. Obesity. 15 (12):2974-83.

Chambers, L., K. McCrickerd, and M.R. Yeomans. (2015). Optimising foods for satiety. Trends in Food Science \& Technology. 41 (2):149-60.

Clegg, M E. (2017). They say coconut oil can aid weight loss, but can it really? European Journal of Clinical Nutrition. 71 (10):1139-43.

Clegg, M E, M. Golsorkhi, and C.J. Henry. (2013). Combined medium-chain triglyceride and chilli feeding increases diet-induced thermogenesis in normal-weight humans. European Journal of Nutrition. 52 (6):1579-85.

Clegg, Miriam E., M. Pratt, O. Markey, et al. (2012). Addition of different fats to a carbohydrate food: impact on gastric emptying, glycaemic and satiety responses and comparison with in vitro digestion. Food Research International. 48 (1):91-97.

Clegg, Miriam E. (2010). Medium-chain triglycerides are advantageous in promoting weight loss although not beneficial to exercise performance. International Journal of Food Sciences and Nutrition. 61 (7):653-79.

Cohen, J. (1992). A power primer. Psychological Bulletin. 112 (1):155-59. 
Coleman, H., P. Quinn, and M.E. Clegg. (2016). Medium chain triglycerides and conjugated linoleic acids in beverage form increase satiety and reduce food intake in humans. Nutrition Research. 36 (6):526-33.

Denke, M.A., and S.M. Grundy. (1992). Comparison of effects of lauric acid and palmitic acid on plasma lipids and lipoproteins. The American Journal of Clinical Nutrition. 56 (5). Oxford University Press:895-98.

Elrick, H., L. Stimmler, C.J. Hlad, et al. (1964). Plasma insulin response to oral and intravenous glucose administration. The Journal of Clinical Endocrinology and Metabolism. 24:1076-82. English, P.J., M.A. Ghatei, I.A. Malik, et al. (2002). Food fails to suppress ghrelin levels in obese humans. The Journal of Clinical Endocrinology \& Metabolism. 87 (6):2984-2984. Feinle, C., T. Rades, B. Otto, et al. (2001). Fat digestion modulates gastrointestinal sensations induced by gastric distention and duodenal lipid in humans. Gastroenterology. 120 (5):11001107.

Feltrin, K.L., T.J. Little, J.H. Meyer, et al. (2004). Effects of intraduodenal fatty acids on appetite, antropyloroduodenal motility, and plasma cck and glp-1 in humans vary with their chain length. American Journal of Physiology. Regulatory, Integrative and Comparative Physiology. 287:R524-33.

Feltrin, K.L., T.J. Little, J.H. Meyer, et al. (2007). Effects of lauric acid on upper gut motility, plasma cholecystokinin and peptide yy, and energy intake are load, but not concentration, dependent in humans. The Journal of Physiology. 581 (2):767-77.

Feltrin, K.L., T.J. Little, J.H. Meyer, et al. (2008). Comparative effects of intraduodenal infusions of lauric and oleic acids on antropyloroduodenal motility, plasma cholecystokinin and peptide yy, appetite, and energy intake in healthy men. The American Journal of Clinical Nutrition. 87 (5):1181-87.

Feltrin, K.L., M. Patterson, M.A. Ghatei, et al. (2006). Effect of fatty acid chain length on 
suppression of ghrelin and stimulation of pyy, glp-2 and pp secretion in healthy men. Peptides. 27 (7):1638-43.

Flint, A., A. Raben, A. Ersbøll, et al. (2001). The effect of physiological levels of glucagon-like peptide-1 on appetite, gastric emptying, energy and substrate metabolism in obesity. International Journal of Obesity. 25 (6):781-92.

Franklin, J.C., B.C. Schiele, J. Brozek, et al. (1948). Observations on human behavior in experimental semi-starvation and rehabilitation. Journal of Clinical Psychology. 4 (1):28-45. French, S.J., C.A. Conlon, S.T. Mutuma, et al. (2000). The effects of intestinal infusion of longchain fatty acids on food intake in humans. Gastroenterology. 119 (4):943-48.

Gibbs, J., R.C. Young, and G.P. Smith. (1973). Cholecystokinin elicits satiety in rats with open gastric fistulas. Nature, UK. 245 (5424):323-25.

Goedecke, J.H., V.R. Clark, T.D. Noakes, et al. (2005). The effects of medium-chain triacylglycerol and carbohydrate ingestion on ultra-endurance exercise performance. International Journal of Sport Nutrition and Exercise Metabolism. 15 (1):15-27.

Hetherington, M.M., K. Cunningham, L. Dye, et al. (2013). Potential benefits of satiety to the consumer: scientific considerations. Nutrition Research Reviews. 26:22-38.

Heymsfield, S.B., J.B. Harp, M.L. Reitman, et al. (2007). Why do obese patients not lose more weight when treated with low-calorie diets? a mechanistic perspective. The American Journal of Clinical Nutrition. 85 (2):346-54.

Higgins, Julian P T, D.G. Altman, P.C. Gøtzsche, et al. (2011). The cochrane collaboration's tool for assessing risk of bias in randomised trials. BMJ (Clinical Research Ed.). 343 (October). British Medical Journal Publishing Group:d5928.

Hill, J.O., H.R. Wyatt, and J.C. Peters. (2012). Energy balance and obesity. Circulation. 126 (1). NIH Public Access:126-32.

Jeukendrup, A.E., J.J. Thielen, A.J. Wagenmakers, et al. (1998). Effect of medium-chain 
triacylglycerol and carbohydrate ingestion during exercise on substrate utilization and subsequent cycling performance. The American Journal of Clinical Nutrition. 67 (3):397-404. Kinsella, R., T. Maher, and M.E. Clegg. (2017). Coconut oil has less satiating properties than medium chain triglyceride oil. Physiology \& Behavior. 179 (October):422-26.

Kissileff, H.R., F.X. Pi-Sunyer, J. Thornton, et al. (1981). C-terminal octapeptide of cholecystokinin decreases food intake in man. The American Journal of Clinical Nutrition. 34 (2):154-60.

Krotkiewski, M. (2001). Value of vlcd supplementation with medium chain triglycerides. International Journal of Obesity and Related Metabolic Disorders. 25 (9):1393-1400.

Laeger, T., C.C. Metges, and B. Kuhla. (2010). Role of $\beta$-hydroxybutyric acid in the central regulation of energy balance. Appetite.

Lean, M.E.J., and D. Malkova. (2016). Altered gut and adipose tissue hormones in overweight and obese individuals: cause or consequence? International Journal of Obesity. 40 (4):622-32. Lindgren, O., R.D. Carr, C.F. Deacon, et al. (2011). Incretin hormone and insulin responses to oral versus intravenous lipid administration in humans. Journal of Clinical Endocrinology and Metabolism. 96 (8):2519-24.

Maher, T., and M.E. Clegg. (2018). Dietary lipids with potential to affect satiety: mechanisms and evidence. Critical Reviews in Food Science and Nutrition. 59 (10). Taylor \& Francis:161944.

Marten, B., M. Pfeuffer, and J. Schrezenmeir. (2006). Medium-chain triglycerides. International Dairy Journal.

Matzinger, D., L. Degen, J. Drewe, et al. (2000). The role of long chain fatty acids in regulating food intake and cholecystokinin release in humans. Gut. 46 (5):688-93.

McLaughlin, J., M. Grazia Lucà, M.N. Jones, et al. (1999). Fatty acid chain length determines cholecystokinin secretion and effect on human gastric motility. Gastroenterology. 116 (1):46-53. 
Moher, D., A. Liberati, J. Tetzlaff, et al. (2009). Preferred reporting items for systematic reviews and meta-analyses: the prisma statement (reprinted from annals of internal medicine). Physical Therapy. 89 (9):873-80.

Näslund, E., P. Grybäck, L. Backman, et al. (1998). Distal small bowel hormones: correlation with fasting antroduodenal motility and gastric emptying. Digestive Diseases and Sciences. $\mathbf{4 3}$ (5):945-52.

National Health Service. (2016). Obesity - nhs.uk. 2016.

Nishi, Y., H. Hiejima, H. Hosoda, et al. (2005). Ingested medium-chain fatty acids are directly utilized for the acyl modification of ghrelin. Endocrinology. 146 (5):2255-64.

Page, K.A., A. Williamson, N. Yu, et al. (2009). Medium-chain fatty acids improve cognitive function in intensively treated type 1 diabetic patients and support in vitro synaptic transmission during acute hypoglycemia. Diabetes. 58:1237-44.

Perry, B., and Y. Wang. (2012). Appetite regulation and weight control: the role of gut hormones. Nutrition and Diabetes. 2 (e26).

Poppitt, S.D., C.M. Strik, A.K.H. MacGibbon, et al. (2010). Fatty acid chain length, postprandial satiety and food intake in lean men. Physiology and Behaviour. 101 (1):161-67.

Reinehr, T., P.J. Enriori, K. Harz, et al. (2006). Pancreatic polypeptide in obese children before and after weight loss. International Journal of Obesity. 30 (10):1476-81.

Rizzo, G., U. Masic, J.A. Harrold, et al. (2016). Coconut and sunflower oil ratios in ice cream influence subsequent food selection and intake. Physiology and Behaviour. 164:40-46.

Rolls, B J, N. Gnizak, A. Summerfelt, et al. (1988). Food intake in dieters and nondieters after a liquid meal containing medium-chain triglycerides. American Journal of Clinical Nutrition. 48 (1):66-71.

Rolls, Barbara J., L.S. Roe, J.S. Meengs, et al. (2004). Increasing the portion size of a sandwich increases energy intake. Journal of the American Dietetic Association. 104 (3):367-72. 
Scharrer, E. (1999). Control of food intake by fatty acid oxidation and ketogenesis. Nutrition (Burbank, Los Angeles County, Calif.). 15 (9):704-14.

St-Onge, M.-P., and P.J.H. Jones. (2002). Physiological effects of medium-chain triglycerides: potential agents in the prevention of obesity. Journal of Nutrition. 132 (3):329-32.

St-Onge, M., B. Mayrsohn, M. O’Keeffe, et al. (2014). Impact of medium and long chain triglycerides consumption on appetite and food intake in overweight men. European Journal of Clinical Nutrition. 68 (10):1134-40.

Sterne, J.A.C., M. Egger, and D. Moher. (2011). Addressing reporting biases. In Cochrane Handbook for Systematic Reviews of Interventions., edited by J. P. T. Higgins and S. Green, 5.1.0. The Cochrane Collaboration.

Stubbs, R.J., and C.G. Harbron. (1996). Covert manipulation of the ratio of medium- to longchain triglycerides in isoenergetically dense diets: effect on food intake in ad libitum feeding men. International Journal of Obesity and Related Metabolic Disorders. 20 (5):435-44. Valente, F.X., F.G. Cândido, L.L. Lopes, et al. (2018). Effects of coconut oil consumption on energy metabolism, cardiometabolic risk markers, and appetitive responses in women with excess body fat. European Journal of Nutrition. 57 (4):1627-37.

Verdich, C., J. Lysga, Ê. Madsen, et al. (2000). Effect of obesity and major weight reduction on gastric emptying. International Journal of Obesity. 24 (7):899-905.

World Health Organisation. (2018). Obesity and overweight. 2018.

Wren, A.M., C.J. Small, H.L. Ward, et al. (2000). The novel hypothalamic peptide ghrelin stimulates food intake and growth hormone secretion. Endocrinology. 141 (11):4325-28. Wymelbeke, V. Van, A. Himaya, J. Louis-Sylvestre, et al. (1998). Influence of medium-chain and long-chain triacylglycerols on the control of food intake in men. American Journal of Clinical Nutrition. 68 (2):226-34.

Wymelbeke, V. Van, J. Louis-Sylvestre, and M. Fantino. (2001). Substrate oxidation and control 
of food intake in men after a fat-substitute meal compared with meals supplemented with an isoenergetic load of carbohydrate, long-chain triacylglycerols, or medium-chain triacylglycerols. American Journal of Clinical Nutrition. 74 (5):620-30.

Yang, N., X. Liu, E.L. Ding, et al. (2009). Impaired ghrelin response after high-fat meals is associated with decreased satiety in obese and lean chinese young adults. The Journal of Nutrition. 139 (7):1286-91.

Zwirska-Korczala, K., S.J. Konturek, M. Sodowski, et al. (2007). Basal and postprandial plasma levels of pyy, ghrelin, cholecystokinin, gastrin and insulin in women with moderate and morbid obesity and metabolic syndrome. Journal of Physiology and Pharmacology : An Official Journal of the Polish Physiological Society. 58 Suppl 1 (March):13-35. 